\title{
A Note on Minimal Galois Embeddings of Abelian Surfaces
}

\author{
HisaO YoSHIHARA
}

ABSTRACT - We show that the least number $N$ such that an abelian surface has a Galois embedding in $\mathbb{P}^{N}$ is seven and then we give examples of such surfaces.

\section{Introduction.}

This is a continuation of our previous paper [7]. The least number $N$ such that an abelian surface can be embedded in $\mathbb{P}^{N}$ is four, and in that case the abelian surface has a special structure, see for example [3]. Similarly it might have some interest to study the least number $N$ such that an abelian surface $A$ can be Galois-embedded in $\mathrm{P}^{N}$. Moreover, in that case we want to know the structure of $A$. In this short note we give the answer to the problem. Before stating it, we recall from [7] some of the definitions and properties of Galois embeddings of algebraic varieties.

Let $k$ be the ground field of our discussions, which is assumed to be algebraic closed. Let $V$ be a nonsingular projective algebraic variety of dimension $n$ and $D$ a very ample divisor. We denote this by a pair $(V, D)$. Let $f=f_{D}: V \hookrightarrow \mathbb{P}^{N}$ be the embedding of $V$ associated with the complete linear system $|D|$, where $N+1=\operatorname{dim} H^{0}(V, \mathcal{O}(D))$. Suppose that $W$ is a linear subvariety of $\mathbb{P}^{N}$ such that $\operatorname{dim} W=N-n-1$ and $W \cap f(V)=\emptyset$. Then consider the projection $W, \pi_{W}: \mathrm{P}^{N}-\rightarrow W_{0}$ with center $W$, where $W_{0}$ is an $n$-dimensional linear subvariety not meeting $W$. The composition $\pi=\pi_{W} \cdot f$ is a surjective morphism from $V$ to $W_{0} \cong \mathbb{P}^{n}$. Let $K=k(V)$ and $K_{0}=k\left(W_{0}\right)$ be the function fields of $V$ and $W_{0}$ respectively. The covering map $\pi$ induces a finite extension of fields $\pi^{*}: K_{0} \hookrightarrow K$ of degree $d=\operatorname{deg} f(V)=D^{n}$, which is the self-intersection number of $D$. It is easy to see that the structure of this extension does not depend on the choice of $W_{0}$

(*) Indirizzo dell'A.: Department of Mathematics, Faculty of Science, Niigata University, Niigata 950-2181, Japan.

E-mail: yosihara@math.sc.niigata-u.ac.jp 
but only on $W$, hence we denote by $K_{W}$ the Galois closure of this extension and by $G_{W}=\operatorname{Gal}\left(K_{W} / K_{0}\right)$ the Galois group of $K_{W} / K_{0}$. Note that $G_{W}$ is isomorphic to the monodromy group of the covering $\pi: V \longrightarrow W_{0}$.

Definition 1. In the above situation we call $G_{W}$ the Galois group at $W$. If the extension $K / K_{0}$ is Galois, we call $f$ and $W$ a Galois embedding and a Galois subspace for the embedding, respectively.

Definition 2. A nonsingular projective algebraic variety $V$ is said to have a Galois embedding if there exist a very ample divisor $D$ such that the embedding associated with the complete linear system $|D|$ has a Galois subspace. In this case the pair $(V, D)$ is said to define a Galois embedding.

In this note we use the following notation.

- $Z_{m}$ : the cyclic group of order $m$

- $D_{m}$ : the dihedral group of order $2 m$

- $|G|$ : the order of a group $G$

- $\rho: \exp (2 \pi \sqrt{-1} / 6)$

- $\operatorname{Aut}(V)$ : the automorphism group of a variety $V$

- $\left\langle a_{1}, \ldots, a_{m}\right\rangle$ : the subgroup generated by $a_{1}, \ldots, a_{m}$

- $\mathbf{1}_{2}$ : the unit matrix of degree two

We shall make use of the following criterion (cf. [7, Theorem 2.2]).

Theorem A. Let $V$ and $D$ be as above. The pair $(V, D)$ defines a Galois embedding if and only if the following conditions hold:

(1) There exists a subgroup $G$ of $A u t(V)$ such that $|G|=D^{n}$.

(2) There exists a G-invariant linear subspace $L$ of $H^{0}(V, \mathcal{O}(D))$ of dimension $n+1$ such that, for any $\sigma \in G$, the restriction $\left.\sigma^{*}\right|_{L}$ is a multiple of the identity.

(3) The linear system L has no base points.

The original form of the study of the Galois embedding is given in [5] or [6]. We have applied the above method to abelian surfaces $A$ over $k=\mathbb{C}$ and obtained some results.

\section{Statement of Theorem.}

Let $A$ be an abelian surface defined over $k=\mathbb{C}$ and $G$ be a finite subgroup of $A u t(A)$. Fix a covering morphism $\mathbb{C}^{2} \longrightarrow A$. An element $g \in G$ has 
a representation $\widetilde{g}$ on the universal covering $\mathbb{C}^{2}$ such that $\widetilde{g} z=M(g) z+t(g)$, where $M(g) \in G L(2, \mathbb{C}), z \in \mathbb{C}^{2}$ and $t(g) \in \mathbb{C}^{2}$. We call $M(g)$ and $t(g)$ the matrix and translation part of the representation $\widetilde{g}$, respectively. Put $G_{0}=\left\{g \in G \mid M(g)=\mathbf{1}_{2}\right\}$ and $H=\{M(g) \mid g \in G\}$. Then, we have the following exact sequence of groups

$$
1 \longrightarrow G_{0} \longrightarrow G \longrightarrow H \longrightarrow 1 \text {. }
$$

Clearly $B=A / G_{0}$ is also an abelian surface and $H \cong G / G_{0}$ is a subgroup of $\operatorname{Aut}(B)$.

Hereafter we assume that $(A, D)$ defines a Galois embedding in $\mathbb{P}^{N}$ and let $G$ be the Galois group. Then $G$ is a subgroup of $A u t(A)$ and $B / H$ is isomorphic to $A / G \cong \mathbb{P}^{2}$. With the notation above, we have:

Theorem B. [7, Theorem 3.7] If an abelian surface A has a Galois embedding, then $H$ is isomorphic to $D_{3}, D_{4}$ or a semidirect product $Z_{2} \times K$, where $K \cong D_{4}$ or $Z_{m} \times Z_{m}(m=3,4,6)$

Now, the answer to the question in the introduction is given as follows:

Theorem C. Suppose that $(A, D)$ defines a Galois embedding. Then

(1) The least number $N$ is seven, i.e., there exists an abelian surface $A$ such that it can be embedded into $\mathrm{P}^{7}$ with a Galois subspace, and no abelian surface embedded in $\mathbb{P}^{N}(N \leq 6)$ has a Galois subspace.

(2) The abelian surface $B=A / G_{0}$ is isomorphic to the self-product $E \times E$ of an elliptic curve, such that $H=G / G_{0}$ acts on $B$ and $H \cong D_{4}$ or $Z_{2} \times D_{4}$.

REMARK 1. The minimal Galois embedding for an elliptic curve $E$ is given as follows. If $E$ can be Galois-embedded in $\mathrm{P}^{2}$, then $E$ must have an automorphism of order three with a fixed point. In fact, the elliptic curve is unique and is defined by $Y^{2} Z=4 X^{3}+Z^{3}$. The centers of the projections are $(1: 0: 0),(0: \sqrt{-3}: 1)$ and $(0:-\sqrt{-3}: 1)$. However, note that every elliptic curve has a Galois embedding in $\mathrm{P}^{3}$, where the group is isomorphic to $Z_{2} \times Z_{2}$ (further, if the $j$-invariant is 1728 , then it has another projection center whose Galois group is isomorphic to $Z_{4}$ ).

In what follows we shall give the proof of Theorem $\mathrm{C}$ and some examples. In particular, there is an example where $B$ is the Jacobian of a curve. Indeed, 
let $J(C)$ be the Jacobian of the normalization $C$ of the curve $y^{2}=$ $x\left(x^{4}+a x^{2}+1\right), a \neq \pm 2$, and let $A$ be an abelian surface which is an etale double covering $q: A \longrightarrow J(C)$. Then we shall show that $\left(A, q^{*}\left(C+C^{\prime}\right)\right)$ gives a minimal Galois embedding, where $C^{\prime}$ is a translated of $C$.

\section{Proof.}

By Theorem B we have $|H|=2^{a} 3^{b}$, where $(a, b)=(3,0),(4,0),(5,0),(1,1)$, $(1,2)(3,2)$. We consider the possible values of $|G|=D^{2}=2 m$. Since $A$ can be embedded in $\mathbb{P}^{m-1}$ by the complete linear system $|D|$, we have $m-1 \geq 4$ by [3]. In view of Theorem B we have $m \neq 5$, hence $m \geq 6$. Suppose that $m=6$. Then, from Theorem B again we infer that $H \cong D_{3}$ and $\left|G_{0}\right|=2$. Every two dimensional complex crystallographic group $G$ with $X / G \cong \mathbb{P}^{2}$ has been classified in [4]. Referring to it, we see that $A$ can be expressed as $A=\mathbb{C}^{2} / \Omega$ such that $\Omega$ is the period matrix

$$
\Omega=\left(\begin{array}{cccc}
-1 & \rho^{2} & -\omega & \rho^{2} \omega \\
1 & \rho & \omega & \rho \omega
\end{array}\right)=\left(\begin{array}{cc}
-1 & \rho^{2} \\
1 & \rho
\end{array}\right)\left(\begin{array}{cccc}
1 & 0 & \omega & 0 \\
0 & 1 & 0 & \omega
\end{array}\right),
$$

where $\omega$ is a complex number with $\Im \omega>0$.

Define four vectors as follows:

$$
v_{1}=\left(\begin{array}{c}
-1 \\
1
\end{array}\right), v_{2}=\left(\begin{array}{c}
\rho^{2} \\
\rho
\end{array}\right), v_{3}=\left(\begin{array}{c}
-\omega \\
\omega
\end{array}\right)=\omega v_{1}, v_{4}=\left(\begin{array}{c}
\rho^{2} \omega \\
\rho \omega
\end{array}\right)=\omega v_{2} .
$$

Let $\mathcal{L}_{A}$ be the lattice in $\mathrm{C}^{2}$ generated by $v_{1}, v_{2}, v_{3}$ and $v_{4}$. Let $g_{1}$ be a generator of $G_{0}$ whose representation is $\widetilde{g}_{1} z=z+e$, where $z, e \in \mathbb{C}^{2}$. Since $g_{1}{ }^{2}$ is the identity on $A$, we have $2 e \in \mathcal{L}_{A}$. Let $\mathcal{L}_{B}$ be the lattice generated by $\mathcal{L}_{A}$ and $e$. Then $B=\mathbb{C}^{2} / \mathcal{L}_{B}=A /\left\langle g_{1}\right\rangle$ is also an abelian surface on which the group $H$ acts. As shown in [4], $H$ is generated by $g_{2}$ and $g_{3}$, whose matrix parts are

$$
M_{2}=M\left(g_{2}\right)=\left(\begin{array}{ll}
0 & 1 \\
1 & 0
\end{array}\right) \text { and } M_{3}=M\left(g_{3}\right)=\left(\begin{array}{cc}
-\rho & 0 \\
0 & \rho^{2}
\end{array}\right)
$$

respectively. Since $2 e \in \mathcal{L}_{A}$, the vector $e$ can be expressed as

$$
e=\frac{1}{2} \sum_{i=1}^{4} n_{i} v_{i},
$$

where $n_{i}=0$ or $1(1 \leq i \leq 4)$. Since $G_{0}$ is a normal subgroup of $G$ and $\left|G_{0}\right|=2, g_{1}$ commutes with each element of $G$. Therefore, we infer that 
$M_{i} e-e \in \mathcal{L}_{A}(i=2,3)$. Indeed we have

$$
M_{2} e-e=\left\{-\left(2 n_{1}+n_{2}\right) v_{1}-\left(2 n_{3}+n_{4}\right) v_{3}\right\} / 2 \in \mathcal{L}_{A} .
$$

Thus we have

$$
\left(-n_{2} v_{1}-n_{4} v_{3}\right) / 2 \in \mathcal{L}_{A}
$$

Similarly, considering $M_{3} e-e$, we have

$$
\left\{-n_{2} v_{1}+\left(n_{1}-n_{2}\right) v_{2}-n_{4} v_{3}+\left(n_{3}-n_{4}\right) v_{4}\right\} / 2 \in \mathcal{L}_{A} .
$$

From (1) we get $n_{2}=n_{4}=0$, hence from (2) we get $n_{1}=n_{3}=0$. Since $e \notin \mathcal{L}_{A}$, this is a contradiction. Thus we have $m \geq 7$. From Theorem $\mathrm{B}$ we infer $|G| \neq 14$, hence we have $m \geq 8$. We conclude that the least number $m$ is 8 by the examples in the next section.

REMARK 2. Referring to [4, Theorem 1], we see that in the case (i) $H \cong D_{4}$ the dimension of the moduli space is 1 , but in the case (ii) $H \cong Z_{2} \ltimes D_{4}$ the dimension is zero.

\section{Examples.}

When we make examples, the following lemma is useful.

LEMMA 3. If $M(g)-\mathbf{1}_{2}$ is a nonsingular matrix for $g \in G$, then we can assume $t(g)=0$.

Proof. We consider $\tau G \tau^{-1}$ instead of $G$, where $\tau$ is a translation $\tilde{\tau} z=z+\ell$. If $M(g)-\mathbf{1}_{2}$ is nonsingular, then by putting $\ell=$ $\left(M(g)-\mathbf{1}_{2}\right)^{-1} t(g)$, we get $t\left(\tau g \tau^{-1}\right)=0$.

In case $m=8$ we have $H \cong D_{4}$ or $Z_{2} \ltimes D_{4}$ by Theorem $\mathrm{B}$. We shall give examples of both. If (i) $H \cong D_{4}$, then $\left|G_{0}\right|=2$. Since $G_{0}$ is a normal subgroup of $G$ and $\left|G_{0}\right|=2$, we infer that $G=G_{0} \times H$. Such examples are given in Examples 4 and 5. On the other hand, if $H \cong Z_{2} \ltimes D_{4}$, we have $G \cong H$. Such an example is given in Example 7 .

ExAmPLE 4. Let $A$ be the abelian surface with the period matrix

$$
\left(\begin{array}{cccc}
1 & 0 & \omega & 0 \\
0 & 1 & 0 & \omega
\end{array}\right) \text { such that } \Im \omega>0 .
$$


Let $\mathcal{L}_{A}$ be the lattice generated by the column vectors of the period matrix. Let us consider the automorphisms $g_{1}, g_{2}$ and $g_{3}$ of $A$, whose representations on $\mathrm{C}^{2}$ are as follows:

$$
\begin{aligned}
\widetilde{g_{1}} z= & z+\frac{1}{2}\left(\begin{array}{l}
n_{1}+n_{3} \omega \\
n_{2}+n_{4} \omega
\end{array}\right), \\
\widetilde{g_{2}} z= & \left(\begin{array}{ll}
0 & 1 \\
1 & 0
\end{array}\right) z+\left(\begin{array}{l}
\alpha_{1} \\
\alpha_{2}
\end{array}\right), \\
\widetilde{g_{3}} z= & \left(\begin{array}{rr}
0 & -1 \\
1 & 0
\end{array}\right) z \\
& \text { where }\left(n_{1}, n_{2}, n_{3}, n_{4}\right)=(0,0,1,1),(1,1,0,0),(1,1,1,1), \\
& \left(\begin{array}{c}
\alpha_{1}+\alpha_{2} \\
\alpha_{1}+\alpha_{2}
\end{array}\right) \in \mathcal{L}_{A} \text { and }\left(\begin{array}{c}
2 \alpha_{1} \\
0
\end{array}\right) \in \mathcal{L}_{A} .
\end{aligned}
$$

We have $g_{1}{ }^{2}=g_{2}{ }^{2}=g_{3}{ }^{4}=i d, g_{2} g_{3} g_{2}=g_{3}{ }^{-1}$ and $g_{i} g_{1}=g_{1} g_{i}(i=2,3)$ on $A$. Putting $G=\left\langle g_{1}, g_{2}, g_{3}\right\rangle$, we have $G_{0}=\left\langle g_{1}\right\rangle$ and $G=G_{0} \times H$ where $H=\left\langle M\left(g_{2}\right), M\left(g_{3}\right)\right\rangle$. Clearly $H \cong D_{4}$. The group $G$ is a subgroup of $A u t(A)$ and $A / G \cong \mathbb{P}^{2}$. The very ample divisor $D$ is given by $\pi^{*}(L)$, where $\pi: A \longrightarrow A / G \cong \mathbb{P}^{2}$ and $L$ is a line in $\mathbb{P}^{2}$ (cf. [7, Lemma 3.5]). We infer from Theorem A that $(A, D)$ defines a Galois embedding.

By [7, Corollary 3.8], if $A$ has a Galois embedding, then the abelian surface $B=A / G_{0}$ is isomorphic to $E \times E$ for some elliptic curve $E$. On the other hand, $E \times E$ can be a Jacobian of a curve for some $E$ (cf. [2]). So one may ask what type of genus 2 curve can give the Jacobian whose double covering has a minimal Galois embedding. Let us consider this question in the next example.

Example 5. Let $\Gamma$ be the curve defined by $y^{2}=x\left(x^{4}+a x^{2}+1\right)$, where we assume $a \neq \pm 2$ (cf. [1, Theorem 4.8]). This curve has a singular point at $\infty$. Let $C$ be the normalization of $\Gamma$. The genus of $C$ is two. Let $\sigma$ and $\tau$ be the birational transformations of $\Gamma$ defined by

$$
\sigma(x)=-x, \sigma(y)=i y \text { and } \tau(x)=1 / x, \tau(y)=y / x^{3}
$$

respectively. Clearly we have $\sigma^{4}=\tau^{2}=i d$ and $\tau \sigma \tau=\sigma^{-1}$. Let $\mathcal{H}$ be the group generated by $\sigma$ and $\tau$. Then we have $\mathcal{H} \cong D_{4}$. This group acts on $C$. Let $\mathrm{C}(x, y)$ be the function field of $C$, where $y^{2}=x\left(x^{4}+a x^{2}+1\right)$. Clearly the invariant field of $\mathbb{C}(x, y)$ by $\sigma$ is $\mathrm{C}\left(x^{2}\right)$. Let $\vartheta_{1}$ and $\vartheta_{2}$ be a basis of holomorphic 1-forms on $C$ induced from $d x / y$ and $x d x / y$ respectively. 
We have

$$
\sigma^{*}\left(\vartheta_{1}\right)=i \vartheta_{1}, \sigma^{*}\left(\vartheta_{2}\right)=-i \vartheta_{2} \text { and } \tau^{*}\left(\vartheta_{1}\right)=-\vartheta_{2}, \tau^{*}\left(\vartheta_{2}\right)=-\vartheta_{1} .
$$

Let $J(C)$ be the Jacobian of $C$. Taking a base point $P \in C$, the Abel-Jacobi map $j_{P}$ is given as $j_{P}: C \longrightarrow J(C)$ such that $j_{P}(Q) \equiv\left(\int_{P}^{Q} \vartheta_{1}, \int_{P}^{Q} \vartheta_{2}\right)$ (modulo the lattice), where $Q \in C$. If $P$ is a fixed point of $\sigma$, then we have

$$
\int_{P}^{\sigma(Q)} \vartheta=\int_{P}^{Q} \sigma^{*}(\vartheta)
$$

for $Q \in C$, where $\vartheta=\vartheta_{1}$ or $\vartheta_{2}$. Similarly if $P^{\prime}$ is a fixed point of $\tau$, then we have

$$
\int_{P^{\prime}}^{\tau\left(Q^{\prime}\right)} \vartheta=\int_{P^{\prime}}^{Q^{\prime}} \tau^{*}(\vartheta),
$$

where $Q^{\prime} \in C$. Note that if $j_{P}: C \longrightarrow J(C)$ is defined with a base point $P$, and $j_{P^{\prime}}: C \longrightarrow J(C)$ is defined with a base point $P^{\prime}$, then $j_{P^{\prime}}=t \cdot j_{P}$, where $t$ is a translation in $J(C)$. Assume that $P$ is a fixed point of $\sigma$. Then, letting $\widetilde{\sigma}$ and $\tilde{\tau}$ be the representations of $\sigma$ and $\tau$ on $\mathbb{C}^{2}$ respectively, we obtain that they can be expressed as $\widetilde{\sigma} z=M(\sigma) z$ and $\tilde{\tau} z=M(\tau) z+v$, where $z \in \mathbb{C}^{2}, v \in \mathbb{C}^{2}$ and

$$
M(\sigma)=\left(\begin{array}{rr}
i & 0 \\
0 & -i
\end{array}\right) \text { and } M(\tau)=\left(\begin{array}{cc}
0 & -1 \\
-1 & 0
\end{array}\right)
$$

Put $H=\langle M(\sigma), M(\tau)\rangle$. Then we have $H \cong D_{4}$. Note that the curve $j_{P}(C)$ is fixed by $\sigma$ and $C /\langle\sigma\rangle$ is isomorphic to a smooth rational curve. We infer from the above arguments that $J(C) / H$ is isomorphic to $\mathrm{P}^{2}$ and $C /\langle\sigma\rangle$ is a line. Let $p: J(C) \longrightarrow J(C) / H \cong \mathbb{P}^{2}$ be the quotient morphism. Then $p^{*}(L)$ can be expressed as $C+C^{\prime}$, where $L$ is the line and $C^{\prime}$ is a translation of $C$ on $J(C)$. Let $A$ be an abelian surface such that $q: A \longrightarrow J(C)$ is an etale double covering given as follows. Express $J(C)=\mathbb{C}^{2} / \mathcal{L}$ and $A=\mathbb{C}^{2} / \mathcal{L}_{0}$, where $\mathcal{L}$ and $\mathcal{L}_{0}$ are lattices satisfying $\left|\mathcal{L}: \mathcal{L}_{0}\right|=2$. Take an element $\ell \in \mathcal{L} \backslash \mathcal{L}_{0}$ so that $\rho(z)=z+\ell$ is a translation of order two on $A$. Then we have $\mathcal{L}=\left\langle\mathcal{L}_{0}, \ell\right\rangle$. Since $2 \ell \in \mathcal{L}_{0}$, we have $M(2 \ell)=2 M(\ell) \in \mathcal{L}_{0}$, where $M \in H$. Hence we infer that $\sigma$ and $\tau$ induce automorphisms on $A$. We use the same 
letter $H$ to denote the group consisting of the elements which are induced from $H$. Let $G$ be the automorphism group on $A$ generated by $H$ and $\rho$. Then put $\pi=p \cdot q: A \longrightarrow A / G \cong \mathbb{P}^{2}$. Since $\operatorname{deg}(\pi)=16 \geq 10$, we see that $\pi^{*}(L)$ is very ample (cf. [7, Lemma 3.5]). From Theorem A we infer that $\left(A, \pi^{*}(L)\right)$ defines a Galois embedding in $\mathbb{P}^{7}$.

Remark 6. Note that $q^{*}(C)=\widetilde{C}$ is irreducible. Because, if not so, then $q^{*}(C)$ can be written as $C_{1}+C_{2}$. We have $\left(q^{*}(C)\right)^{2}=4$ and $C_{1}^{2}=C_{2}^{2}=2$. Since $C_{1}$ is ample, we have $\left(C_{1}, C_{2}\right) \geq 1$. This is a contradiction. Similarly $q^{*}\left(C^{\prime}\right)=\widetilde{C}^{\prime}$ is also irreducible. The divisor $\widetilde{C}+\widetilde{C}^{\prime}$ gives the minimal Galois embedding of $A$. If $L$ is the image of $C$, then $\widetilde{C}+\widetilde{C}^{\prime}=\pi^{*}(L)$.

ExAmPLE 7. Let $A$ be the abelian surface with period matrix

$$
\left(\begin{array}{cccc}
1 & 0 & i & (1+i) / 2 \\
0 & 1 & 0 & (1+i) / 2
\end{array}\right)
$$

This abelian surface has the automorphisms $g_{1}, g_{2}$ and $g_{3}$, whose representations on $\mathbb{C}^{2}$ are as follows:

$$
\begin{aligned}
& \widetilde{g_{1} z}=\left(\begin{array}{rr}
-1 & 0 \\
0 & 1
\end{array}\right) z+\left(\begin{array}{l}
e_{11} \\
e_{12}
\end{array}\right), \\
& \widetilde{g_{2}} z=\left(\begin{array}{ll}
0 & 1 \\
1 & 0
\end{array}\right) z+\left(\begin{array}{l}
e_{21} \\
e_{22}
\end{array}\right), \\
& \widetilde{g_{3}} z=\left(\begin{array}{rr}
i & 0 \\
0 & -i
\end{array}\right) z,
\end{aligned}
$$

where the following vectors belong to the lattice generated by the column vectors of the period matrix:

$$
\begin{array}{cc}
\left(\begin{array}{c}
0 \\
2 e_{12}
\end{array}\right), \quad\left(\begin{array}{c}
e_{21}+e_{22} \\
e_{21}+e_{22}
\end{array}\right), & \left(\begin{array}{c}
e_{11}-e_{12}-2 e_{21} \\
e_{11}+e_{12}
\end{array}\right), \\
\left(\begin{array}{c}
(1-i) e_{11} \\
(1-i) e_{12}
\end{array}\right), & \left(\begin{array}{c}
e_{21}-i e_{22} \\
e_{22}+i e_{21}
\end{array}\right) .
\end{array}
$$

We have $g_{1}{ }^{2}=g_{2}{ }^{2}=g_{3}{ }^{4}=i d, g_{1} g_{2} g_{1}=g_{2} g_{3}^{2}, g_{1} g_{3} g_{1}=g_{3}$ and $g_{2} g_{3} g_{2}=g_{3}{ }^{-1}$. Putting $G=\left\langle g_{1}, g_{2}, g_{3}\right\rangle$, we see that $G$ is isomorphic to the semidirect product $Z_{2} \ltimes D_{4}$ and $G$ is a subgroup of $A u t(A)$ and $A / G \cong \mathbb{P}^{2}$. The very ample divisor $D$ is given by $\pi^{*}(L)$, where $\pi: A \longrightarrow A / G \cong \mathbb{P}^{2}$ and $L$ is a line in $\mathbb{P}^{2}$. We infer from Theorem $\mathrm{A}$ that $(A, D)$ defines a Galois embedding. 
REMARK 8. The abelian surface $A$ in Example 7 is isogenous to $E_{i} \times E_{i}$, where $E_{i}=\mathrm{C} /(1, i)$. (In fact, we can show that $A$ is isomorphic to $E_{i} \times E_{i}$. ) Thanks to [2], this abelian surface cannot be a Jacobian of a curve.

\section{REFERENCES}

[1] A. FuJIKI, Finite automorphism groups of complex tori of dimension two, Publ. RIMS. Kyoto Univ., 24 (1988), pp. 1-97.

[2] T. HAYASHIDA - M. Nishi, Existence of curves of genus two on a product of two elliptic curves, J. Math. Soc. Japan, 17 (1965), pp. 1-16.

[3] K. HuleK - H. LANGe, Examples of abelian surfaces in $\mathbb{P}^{4}$, Journal Reine Angew. Math., 363 (1985), pp. 200-216.

[4] J. Kaneko - S. TokUnagA - M. Yoshida, Complex crystallographic groups II, J. Math. Soc. Japan, 34 (1982), pp. 595-605.

[5] K. Miura - H. Yoshinara, Field theory for function fields of plane quartic curves, J. Algebra, 226 (2000), pp. 283-294.

[6] H. Yoshinara, Function field theory of plane curves by dual curves, J. Algebra, 239 (2001), pp. 340-355.

[7] H. Yoshinara, Galois embedding of algebraic variety and its application to abelian surface, Rend. Sem. Mat. Universita di Padova, 117 (2007), pp. 69-85.

Manoscritto pervenuto in redazione il 14 aprile 2010. 